\title{
Management of chylous ascites following hand assisted laparoscopic donor
}

\author{
nephrectomy: Case report
}

George Crome, Jacob A Akoh

Department of Surgery, Plymouth Hospitals NHS Trust, Derriford Hospital, Plymouth PL6 8DH, United Kingdom

\section{CASE STUDY}

Please cite this paper as: Crome G, Akoh JA. Management of chylous ascites following hand assisted laparoscopic donor nephrectomy: Case report. AMJ 2017;10(10):894-897.

https://doi.org/10.21767/AMJ.2017.3126

\section{Corresponding Author:}

Mr Jacob A Akoh

Plymouth Hospitals NHS Trust, Level 04 Derriford Hospital, Plymouth PL6 8DH, United Kingdom

Tel: +441752432650

Email: Jacob.akoh@nhs.net

\section{ABSTRACT}

We report a case of chylous ascites following a hand assisted laparoscopic donor nephrectomy (HALDN). In this case a 70-year-old male was re-admitted three weeks after undergoing a left HALDN with anorexia and abdominal distension. Abdominal CT confirmed ascites. Paracentesis yielded five litres of chylous fluid. He was treated conservatively with a high protein, low fat diet and made a full recovery. The management of this complication remains controversial with some advocating early surgical intervention and others preferring conservative management techniques. Consideration must be given to early surgical intervention in cases not responding immediately to conservative measures.

\section{Key Words}

Laparoscopic donor nephrectomy, chylous ascites, conservative management

\section{Implications for Practice:}

\section{What is known about this subject?}

Chyle leak is a rare complication of LDN due to damage of the lymphatic structures causing accumulation of chyle in the abdominal cavity reported only following left nephrectomy.

\section{What new information is offered in this case study?}

A late phase contrast CT scan may eliminate urine leak as a differential diagnosis by showing no extravasation of contrast from the bladder.

3. What are the implications for research, policy, or practice?

Dye injection in the lower extremity may identify patients at risk during nephrectomy as the dye may leak into the operating field, thereby allowing timely diagnosis and clipping of damaged lymph channels?

\section{Background}

Since its introduction in 1995, laparoscopic donor nephrectomy (LDN) has become the standard technique for kidney removal. Not only does it have similar graft success rates compared to open nephrectomy but it is associated with faster postoperative recovery and decreased postoperative pain. ${ }^{1}$ Though the technique has evolved, the introduction of a hand port has remained popular as it combines the manual dexterity associated with open surgery with the improved access and views available laparoscopically. ${ }^{2}$ Despite the benefits of LDN, there are also some complications that may occur with LDN, which rarely occur during open procedures. One such rare complication of LDN is chyle leak due to damage of the lymphatic structures causing accumulation of chyle in the abdominal cavity (chylous ascites). We present a case of chylous ascites in a 70-year-old male following hand assisted laparoscopic left donor nephrectomy (HALDN) that was successfully managed conservatively and review the literature.

\section{Case details}

A 70-year-old male had his left kidney recovered by means of a HALDN. Prior to this, he was fully assessed as per the protocol of the South West Transplant Centre and found 
suitable to donate his left kidney. His past medical history included open tonsillectomy; appendicectomy; open cholecystectomy; Ludwig's angina and a herniated disc. He underwent cardiopulmonary exercise testing showing good cardiorespiratory reserves indicating a low risk for postoperative complications.

At surgery, he was found to have a large left kidney. The ureter was dissected. Dissection was performed using the LOTUS $^{\mathrm{TM}}$ ultrasonic device. The gonadal vein was exposed and followed to expose the renal vein. The suprarenal and gonadal veins were divided using the LigaSure ${ }^{\mathrm{TM}}$ bipolar sealing device. The ureter was liga-clipped at the pelvic brim and the kidney retrieved after division of the renal artery and vein. He did well and was discharged home five days postoperatively.

He was re-admitted approximately three weeks later with increasing abdominal discomfort, bloating, weight loss and poor appetite. On examination, his abdomen was soft with ascites present. He exhibited deranged liver function tests alkaline phosphatase of $260 \mathrm{IU} / \mathrm{L}$ and alanine transaminase of 72IU/L. His C-reactive protein (CRP) and white cell count (WCC) which were $42 \mathrm{mg} / \mathrm{L}$ and $7.9 \times 10^{9} / \mathrm{L}$ respectively at discharge had risen to $128 \mathrm{mg} / \mathrm{L}$ and $8.8 \times 10^{9} / \mathrm{L}$ respectively on re-admission. A contrast enhanced computed tomography (CT) scan of the abdomen and pelvis (Figure 1) showed a large amount of free fluid of high attenuation in the abdomen. There was no leak of contrast from the left ureteric stump.

An ascitic tap yielded fluid which on analysis contained pus cells but sterile on culture. His serum and fluid triglyceride levels were similar at $14.3 \mathrm{mmol} / \mathrm{L}$ and $13.3 \mathrm{mmol} / \mathrm{L}$ respectively. He was started on a low fat, high protein diet. Under Ultrasound control, a size $8 \mathrm{Fr}$ pigtail drain was inserted into the peritoneal cavity and about five litres of chylous fluid aspirated. Over the following 3-4 days, the rate of drainage reduced from about $50 \mathrm{ml} /$ hour to $<30 \mathrm{ml} /$ day and the drain was removed seven days after insertion. His albumin reached its lowest two days after drain insertion at $34 \mathrm{~g} / \mathrm{L}$ but promptly recovered. When seen in the follow up clinic six weeks later, he was doing well and had no complaints. There was no evidence of reaccumulation of his ascites and his liver function tests had returned to normal.

\section{Discussion}

Chylous ascites is a rare complication most commonly associated with abdominal aortic surgery and urooncological surgery due to the need for lymph node dissection. ${ }^{3}$ There is a growing number of reports of chylous ascites following donor nephrectomy, with the majority of these occurring after laparoscopic procedures. On review of the literature, we found a total of 57 cases of chylous ascites $^{4-8}$ of which two were associated with open procedures ${ }^{6}$ and one following robot assisted LDN. ${ }^{4}$ It is interesting to note that all the reports of chylous ascites identified were associated with left laparoscopic nephrectomy. Meulen et al. ${ }^{5}$ hypothesise that the proximity of ligation of the renal artery to the aorta may be the cause for this. The major lymphatic vessels lie in the para aortic area and are liable to be disrupted when dissecting the renal artery close to the aorta - often done to achieve enough length of the donated renal artery. On the right the ligation is latero-caudal to the inferior vena cava and therefore much further from the para aortic lymphatics. ${ }^{5}$ One other possible explanation could be a surgical preference for the left kidney, possibly due to the long length of the renal vein on the left. This raises the question whether the total number of right LDN are probably too few to identify chyle leak. The lack of a single report linking chylous ascites with right donor nephrectomy makes this an unlikely explanation.

A number of considerations are important in trying to prevent the development of chylous ascites. Firstly, awareness of this complication may help surgeons to take steps to prevent it. Secondly, the use of energy sealing devices such as LigaSure ${ }^{\mathrm{TM}}$ may effect better and more efficient sealing of the para-aortic lymphatics and reduce the chance of lymphatic leak from the renal bed. Thirdly, close visualisation of the renal bed immediately after removal of the kidney may reveal early lymphatic leak of creamy white discharge of fluid around the aortic stump which can be ligated before the wound is closed.

Chylous ascites can present a range of different challenges for the treating team including significant nutritional deficit as well as immunocompromise following lymphatic losses and direct pressure effects from ascites causing splinting of the diaphragm and respiratory complications. ${ }^{9}$ The diagnosis can be made following paracentesis with the production of a milky fluid which on analysis has a 2 to 8 fold increase in triglyceride levels from the serum as well as a high protein level. Serum abnormalities may include a lymphocytosis, hypoalbuminaemia and anaemia., ${ }^{3,9}$ In our case the triglyceride levels were very similar and one explanation for this could be due to the nutritional state at the time of testing, as he had already been started on the modified diet. ${ }^{3}$ CT imaging is not specific for chylous ascites as chyle has the same density as urine, bile and simple 
ascites. $^{3}$ A late phase contrast CT scan may eliminate urine leak as a differential diagnosis by showing no extravasation of contrast from the bladder, as in this case. In some cases a fluid level can be seen between the lower density lipids in the chyle, separating from the serous component, making a visible layer on CT scan. This was not the case with our patient possibly due to the lower than usual difference in serum and fluid triglyceride levels. ${ }^{9}$

Although the number of cases of chylous ascites are increasing, no clear guidelines exist for its management. The majority of postoperative chylous leaks can be managed conservatively with paracentesis to relieve symptoms related to abdominal distension and pressure. This can be combined with medium chain fatty acids and high protein diet in the first instance, ${ }^{3}$ as in our case. This diet aims to decrease the amount and flow of chyle allowing any leaks to close. Somatostatin analogues have also been used to control or diminish ascites but the mechanism behind this is still not fully understood. Though total parenteral nutrition (TPN) is often considered a second line of treatment if dietary modification fails, some studies have suggested that early TPN may lead to faster resolution. ${ }^{3}$ Jairath et al. ${ }^{10}$ have proposed a management protocol based on either output per preplaced drain post nephrectomy or response within 48 hours to paracentesis. It is reasonable to hypothesize that those cases that respond to conservative management are those that the lymphatic leak had sealed spontaneously at the time of presentation. While those with continuing leakage of lymphatics at the time of presentation are those that will require surgical intervention aimed at ligating the persisting leaking lymphatics. It is therefore important to identify this later group early and plan surgical treatment promptly.

The role and timing of surgical intervention is evolving. Aerts et al. ${ }^{11}$ looked at a case series of 18 patients following LDN and found that seven (39 per cent) required surgical exploration to resolve leak and of these 86 per cent were successful. The remaining patient required a further prolonged course of TPN. The surgical techniques include facilitating identification of the leak by giving a high fat diet to encourage chyle flow preoperatively as high fat diet make chyle milky cloudy colour instead of the normal colourless colour which is difficult to see during surgery; the ligation and clipping of defects; and sealing using fibrin glue. ${ }^{3,11,12}$ The usual time to perform a repair of refractory chyle leak is between 4-12 weeks postoperatively. ${ }^{3}$ It is conceivable that early intervention will help to avoid the debilitating nutritional and immunological consequences of prolonged conservative treatment that increases the risk of surgical site infections and poor wound healing. It has also been suggested that the source of leakage is easier to identify before partial healing has obscured it. ${ }^{7}$ The optimal time frame to perform surgical repair or identify which patients are at risk of failure to respond to conservative management still remains unclear. Conservative management is likely to be successful in patients with small to moderate output of ascetic fluid per drain. Patients with large output of chylous fluid $(>1000 \mathrm{~mL} /$ day) or with reaccumulation within 48 hours of paracentesis should be considered for lymphography. A positive lymphography performed before treatment in those at risk of continuing leakage may be helpful to initiate early surgical management to avoid debilitation of this condition.

\section{Conclusion}

Though the author's case responded promptly to conservative management, chylous ascites remains a rare but debilitating complication following laparoscopic donor nephrectomy. Might dye injection in the lower extremity identify patients at risk during LDN thereby allowing timely diagnosis and clipping or suturing of damaged lymph channels? Failing such manoeuvre, early surgical intervention might prevent prolonged debilitation in these donors.

\section{References}

1. Shokier A. Open versus laparoscopic live donor nephrectomy: A focus on the safety of donors and the need for a donor registry. J Urol. 2007;178(5):1860-6. doi: 10.1016/j.juro.2007.07.008

2. Dols LF, Kok NF, d'Ancona FC, et al. Randomized controlled trial comparing hand-assisted retroperitoneoscopic versus standard laparoscopic donor nephrectomy. Transplantation. 2014;97(2):161-7. doi: 10.1097/TP.0b013e3182a902bd.

3. Kim BS, Kwon TG. Chylous ascites in laparoscopic renal surgery: Where do we stand? World J Clin Urol. 2016;5(1):37-44. doi: 10.5410/wjcu.v5.i1.37

4. Janki S, Terkivatan $\mathrm{T}$, Kimenai $\mathrm{H}$, et al. First case report of chylous ascites after robot-assisted donor nephrectomy. J Surg Case Reports. 2016;7. doi: 10.1093/jscr/rjw118

5. Meulen ST, van Donselaar-van der Pant KA, Bemelman FJ, et al. Chylous ascites after laparoscopic hand-assisted donor nephrectomy: Is it specific for the left-side? Urol Ann. 2013;5(1):45-46. doi:10.4103/0974-7796.106967.

6. Harkar S, Singh DV, Gupta SK, et al. Chyle Leak following Open Donor Nephrectomy: A Rare Complication - A Case Report. Case Rep Transplant. 2012;2012:259838. doi:10.1155/2012/259838. 
7. Caumartin Y, Pouliot F, Sabbagh R, et al. Chylous ascites as a complication of laparoscopic donor nephrectomy. Transplant Int. 2005;18:1378-1381. doi:10.1111/j.14322277.2005.00219.x

8. Molina WR, Desai MM, Gill IS. Laparoscopic management of chylous ascites after donor nephrectomy. J Urol. 2003;170(5):1938. doi: 10.1097/01.ju.0000091660.15018.66

9. Shah SS, Ahmed K, Smith R, et al. Chylous ascites following radical nephrectomy: a case report. J Med Case Reports. 2008;2:3. doi: 10.1186/1752-1947-2-3.

10. Jairath A, Singh A, Ganpule A, et al. Management protocol for chylous ascites after laparoscopic nephrectomy. Urol. 2015;86(3):521-8.

11. Aerts J, Matas A, Sutherland D, et al. Chylous ascites requiring surgical intervention after donor nephrectomy - Case series and single center experience. Am J Transplant. 2010;10(1):124-128. doi:10.1111/j.16006143.2009.02883.x.

12. Nakamura $Y$, Fujisawa $Y$, Maruyama $H$, et al. Interoperative mapping with isosulfan blue of lymphatic leakage during inguinal lymph node dissection for skin cancer for the prevention of postoperative lymphocele. J Surg Oncol. 2011;104(6):657-60. doi: $10.1002 /$ jso. 21989 .

\section{PEER REVIEW}

Not commissioned. Externally peer reviewed.

\section{CONFLICTS OF INTEREST}

The authors declare that they have no competing interests.

\section{FUNDING}

None

\section{PATIENT CONSENT}

The authors, Crome $G$ and $A k o h J A$, declare that:

1. They have obtained written, informed consent for the publication of the details relating to the patient(s) in this report.

2. All possible steps have been taken to safeguard the identity of the patient(s).

3. This submission is compliant with the requirements of local research ethics committees.
Figure 1: Abdominal CT scan revealing absent left kidney and a large intra-abdominal fluid collection

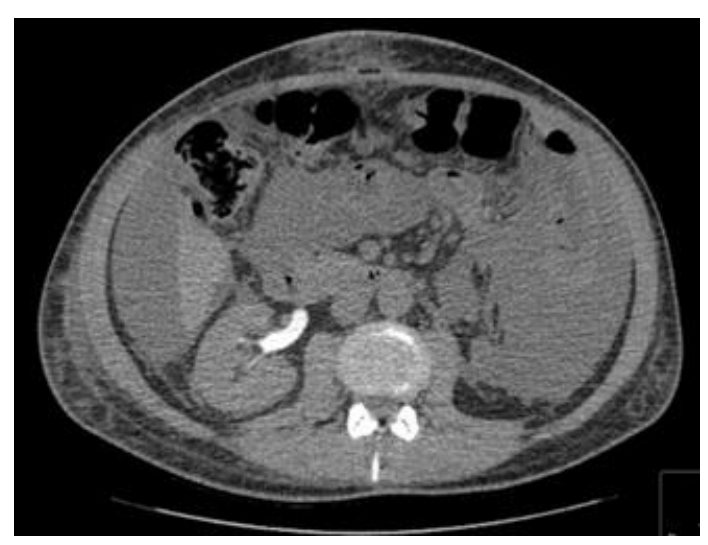

